\title{
Pictorial Depiction of an Institutional Experience of Acute Abdominal Emergencies in Cancer Patients with COVID-19 Infection
}

\author{
Ankush Jajodia ${ }^{1}$ Anuraag Jena ${ }^{2} \quad$ Sunil Pasricha $^{3} \quad$ Shaifali Goel $^{4} \quad$ Gurudutt Gupta $^{3}$ Sunil Puri ${ }^{1}$ \\ ${ }^{1}$ Department of Radiology, Rajiv Gandhi Cancer Institute and \\ Research Centre, Delhi, India \\ 2 Department of Gastroenterology, Postgraduate Institute of Medical \\ Education and Research, Chandigarh, India \\ ${ }^{3}$ Department of Histopathology, Rajiv Gandhi Cancer Institute, Delhi, \\ India \\ ${ }^{4}$ Department of Surgical Oncology, Rajiv Gandhi Cancer Institute, \\ Delhi, India \\ J Gastrointestinal Abdominal Radiol ISGAR 2022;5:165-170. \\ Address for correspondence Ankush Jajodia, DMRD, DNB, MNAMS, \\ Department of Radiology, Rajiv Gandhi Cancer Institute and Research \\ Centre, Rohini Sector 5, Delhi 110085, India \\ (e-mail: ankushjaj@gmail.com).
}

\begin{abstract}
The ongoing pandemic of coronavirus disease 2019 (COVID-19) poses a diagnostic and management challenge to the clinician, which further gets heightened when patients present with abdominal complications in a cancer care center. The atypical manifestations of the disease provide a diagnostic conundrum to the radiologist and leave the pathologist in the perils of guiding further management to the clinician. Although previous literature shows gastrointestinal involvement in COVID-19, we present a case

Keywords

- COVID-19

- cancer patients

- gastrointestinal

- computed tomography series of complicated subjects with clinical imaging as a pictorial essay and relevant pathology. The abdominal manifestations of COVID-19 are complicated in cancer patients where a variety of other differentials such as infiltration by metastatic disease and drug-related chemo toxicity effects must be taken into account, which may be ruled out by clinical workup, adequate imaging, and laboratory tests. This would help achieve better clinical acumen and modify management in these subjects.
\end{abstract}

\section{Introduction}

The acute form of pulmonary illness, which originated from the Wuhan in China, was caused by the novel coronavirus, named coronavirus disease 2019 (COVID-19), and was declared a global emergency and pandemic by the World Health Organization. ${ }^{1}$ The symptoms manifested by this disease did not limit themselves only to the respiratory tract, with the literature showing a plethora of gastrointestinal (GI) manifestations, few amounting presentations as an acute abdomen. ${ }^{2} \mathrm{GI}$ involvement in COVID-19 has been seen in up to $9.8 \%$ of cases, while in $30 \%$ of cases, viral RNA has been detected in fecal samples. The most common GI manifestations include diarrhea (10.4\%), nausea/vomiting (7.7\%), and abdominal discomfort (6.9\%). ${ }^{3}$ Additionally, exacerbation of underlying inflammatory bowel diseases and few instances of GI bleeding have also been reported. ${ }^{4}$ We report a case series of four subjects presenting to our institution (tertiary cancer care center) with either a known history of malignancy with routine surveillance or a new suspicion of published online February 9, 2022
DOI https://doi.org/ $10.1055 / \mathrm{s}-0042-1742678$. ISSN 2581-9933.
(C) 2022. Indian Society of Gastrointestinal and Abdominal Radiology. All rights reserved.

This is an open access article published by Thieme under the terms of the Creative Commons Attribution-NonDerivative-NonCommercial-License, permitting copying and reproduction so long as the original work is given appropriate credit. Contents may not be used for commercial purposes, or adapted, remixed, transformed or built upon. (https://creativecommons.org/ licenses/by-nc-nd/4.0/)

Thieme Medical and Scientific Publishers Pvt. Ltd., A-12, 2nd Floor, Sector 2, Noida-201301 UP, India 
malignancy, who presented to the emergency with an acute abdomen.

\section{Case 1}

A 45-year female diagnosed with squamous cell carcinoma of the cervix, treated with chemoradiation with a radical intent in 2003, presented with a history of abdominal pain. She underwent a routine complete blood count test, abdominopelvic ultrasound, and a colonoscopy at the discretion of the treating radiation oncologist, which were within normal limits. On sudden worsening of her condition within 10 days of the initial complaint, she required admission to the emergency with symptoms of acute abdomen. She had no underlying co-morbidities (diabetes, hypertension, renal, or cardiac medical problems) and no previous history of bowel diseases, and underlying infectious etiology (typhlitis, tuberculosis, and amoebiasis) or radiation-induced enteritis was ruled out during her earlier visit. According to the hospital protocol, upon presentation to the emergency, a nasal swab showed COVID-19 infection by the standard reverse transcriptase-polymerase chain reaction (RT-PCR) test. The hemogram picture revealed normal total leukocyte counts $(6,110$ /cumm) with a lymphopenic picture (6.9\%). Computed tomography (CT) examination ordered showed free air in the peritoneal cavity with a thickened terminal ileal loop lacking regular mural enhancement and pneumatosis coli (-Fig. 1). The solid organs in the abdominal cavity were unremarkable, but lung bases included in the abdominal CT study showed few ground-glass haziness with fibroatelectatic bands. She underwent emergency laparotomy, which showed necrotic changes in the bowel wall culminating in a segmental ileal resection with end-to-end anastomosis. The gross pathological specimen showed edematous mucosa with a perforated area on the external surface, with gray-white surface exudates. On microscopic examination, sections showed focally ulcerated ileal mucosa, edematous areas in submucosa, and acute and chronic lymphoid-plasma cell infiltrates with congested blood vessels. The serosal surface showed acute inflammatory infiltrate and fibrinous material (-Fig. 2).

\section{Case 2}

A 28 years male, diagnosed with axillary epithelioid sarcoma in 2018 and treated with chemoradiation with palliative intent, presented with new-onset abdominal pain and dyspnea amounting to emergency admission. He had no underlying co-morbidities, non-smoker with no previous history of bowel diseases or underlying infectious etiology. Contrast CT angiographic examination showed no free intraperitoneal air with alternate collapsed and prominent large bowel loops showing non-enhancing mucosa and pericolic fluid, indicating intestinal ischemia. No vascular thrombosis was observed and lung bases revealed normal lung parenchyma (-Fig. 3). Surgery was withheld as the patient did not provide consent. Conservative management was done in our institute, and a nasal swab done at the time of admission confirmed COVID-19 infection by the RT-PCR. Hemogram revealed normal total leukocyte counts $(7,820 / \mathrm{cumm})$ with a lymphopenic picture (2.8\%). D-dimers were elevated (617 $\mathrm{ng} / \mathrm{mL}$ ), and CT pulmonary angiography study done 3 days

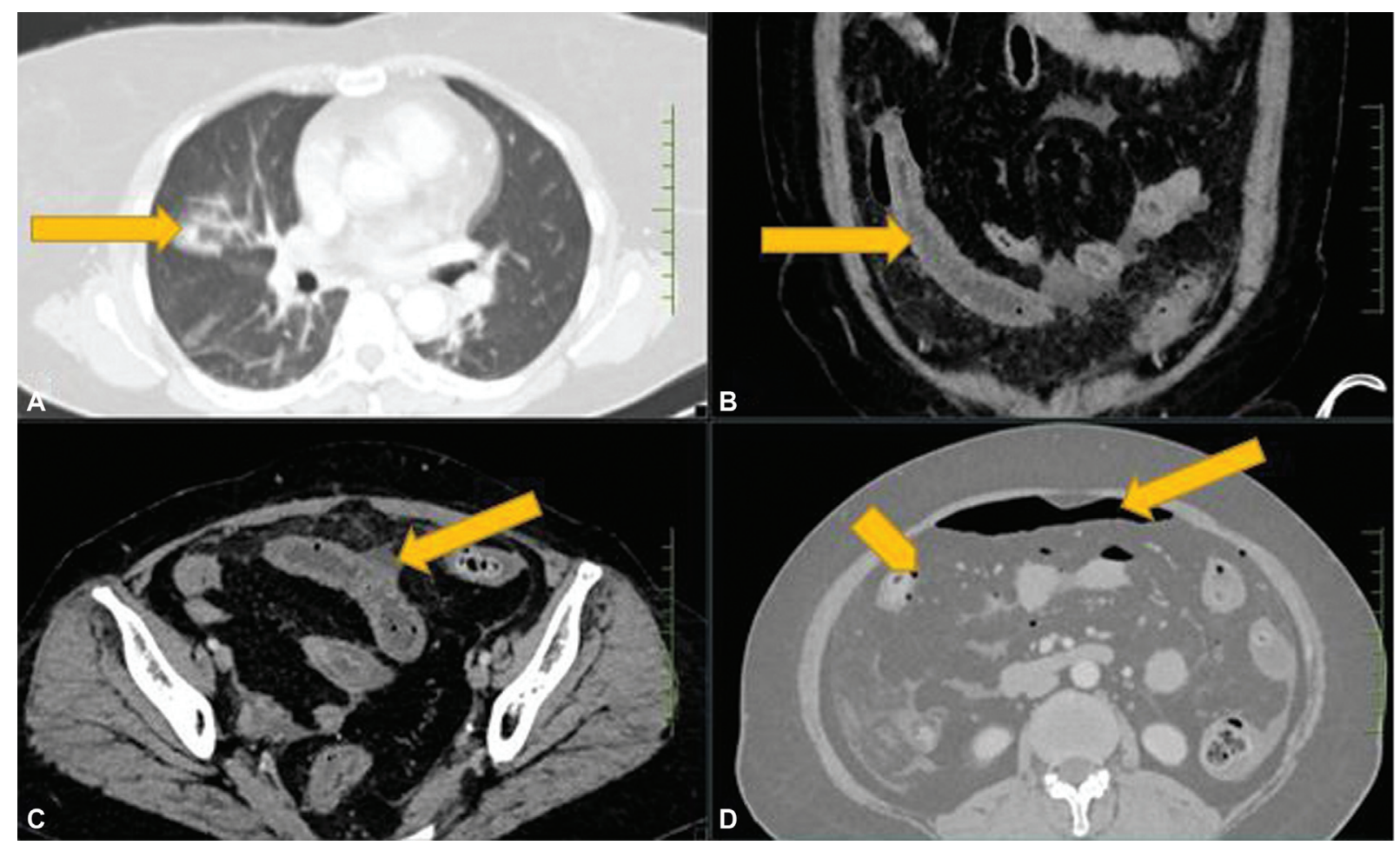

Fig. 1 (A) Axial computed tomography section of case 1 lung window shows ground-glass reticulations. (B and C) Axial and coronal sections in soft tissue window show edematous thickening ileal loop with mesentery haziness and pronounced loco-regional fat stranding and nodularity. (D) Wide window view of contrast axial section obtained to show free intraperitoneal air and pneumatosis in bowel wall. 


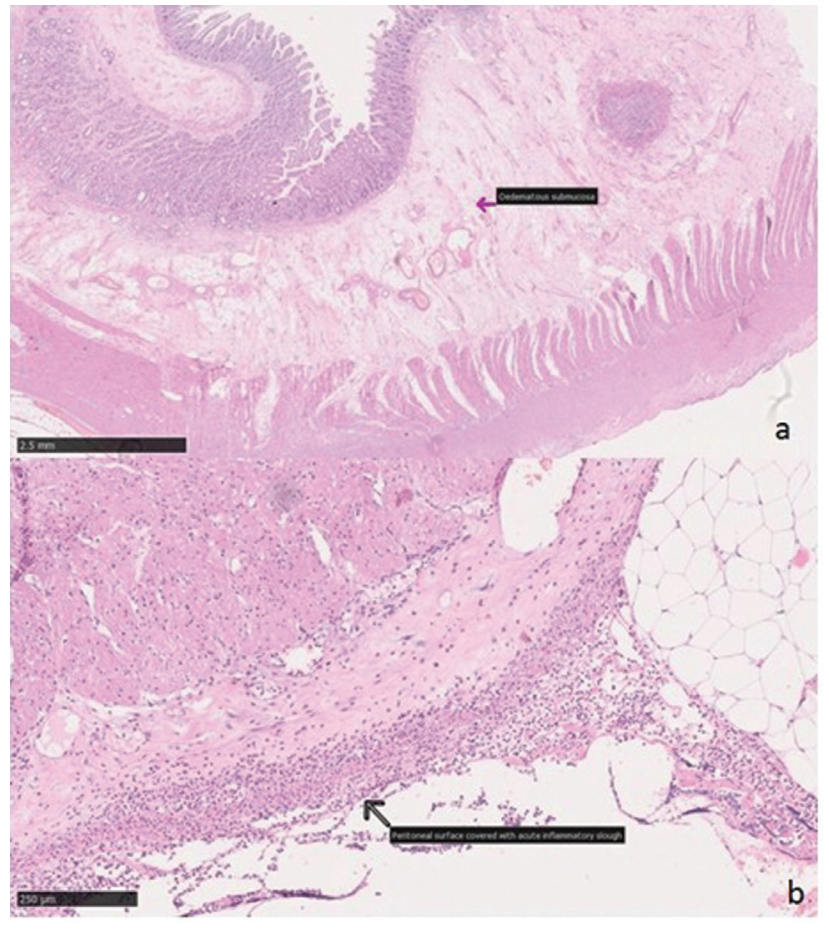

Fig. 2 Histopathological study of case 1 done in routine hematoxylin and eosin stain and viewed under 10X resolution shows acute inflammatory slough on the peritoneal surface (arrow and labeled on slide).

later showed thrombus in segmental pulmonary vessels with a sub-pleural distribution of ground-glass opacities (-Fig. 4). The patient succumbed to complicated pulmonary acute respiratory distress syndrome on the 10th day of admission.

\section{Case 3}

A 42-year male with right iliac fossa pain newly diagnosed as caecal growth amounting to adenocarcinoma on a colonoscopic biopsy done elsewhere presented to our institution with acute abdomen seeking admission. Contrast CT abdomen showed asymmetric circumferential heterogeneously enhancing wall thickening in ileocecal (IC) junction with adjacent paracolic fat stranding and multiple mesenteric nodes. Lower sections of the chest revealed subtle ground-glass opacities in the periphery of the lung parenchyma (-Fig. 5), and nasal swab showed evidence of COVID 19 infection by the standard of RT-PCR test. Subsequently, colonoscopy biopsy done in our institution revealed surface ulceration with the formation of granulation tissue, dense acute on chronic inflammatory cell infiltrate with foci of cryptitis, and crypt abscess in the lamina with no evidence of dysplasia or malignancy. No evidence of granulomas or amoebic trophozoites was seen either (-Fig. 6). The patient improved with conservative management with remdesvir, azithromycin, and favipiravir and was discharged on the 14th day of admission. Repeat RT-PCR while discharge was negative for COVID-19, and CT examination showed mild mesenteric fat stranding with no other abdominal abnormalities.

\section{Case 4}

A 52-year female diagnosed case of breast cancer treated with surgery and adjuvant chemoradiation in 2015 presented severe abdominal pain to the emergency, with guarding and rigidity. Contrast CT study obtained showed prominent bowel loops with small bowel faces sign in ileal loops with a dilated cecum and mild ascites (- Figs. 7 and 8). Lower thoracic sections revealed pleural effusion with patchy ground-glass infiltrates (-Fig. 9). The patient was on regular follow-up, and the recent CT examination ordered a month back had shown no significant abdominal abnormality. The nasal swab showed evidence of COVID-19 infection by the standard RT-PCR test. Routine laboratory investigations for other infectious etiology turned out to be negative. Colonoscopy could not elicit the IC junction abnormality as the scope could not be negotiated beyond a tight structure at the splenic flexure. The procedure was abandoned, and the patient was put on conservative management. Repeat RT-PCR testing and CT examination after 10 days with empirical therapy for COVID-19 showed a negative RT-PCR result and subsidence of the bowel abnormalities as well as ascites on the CT examination.

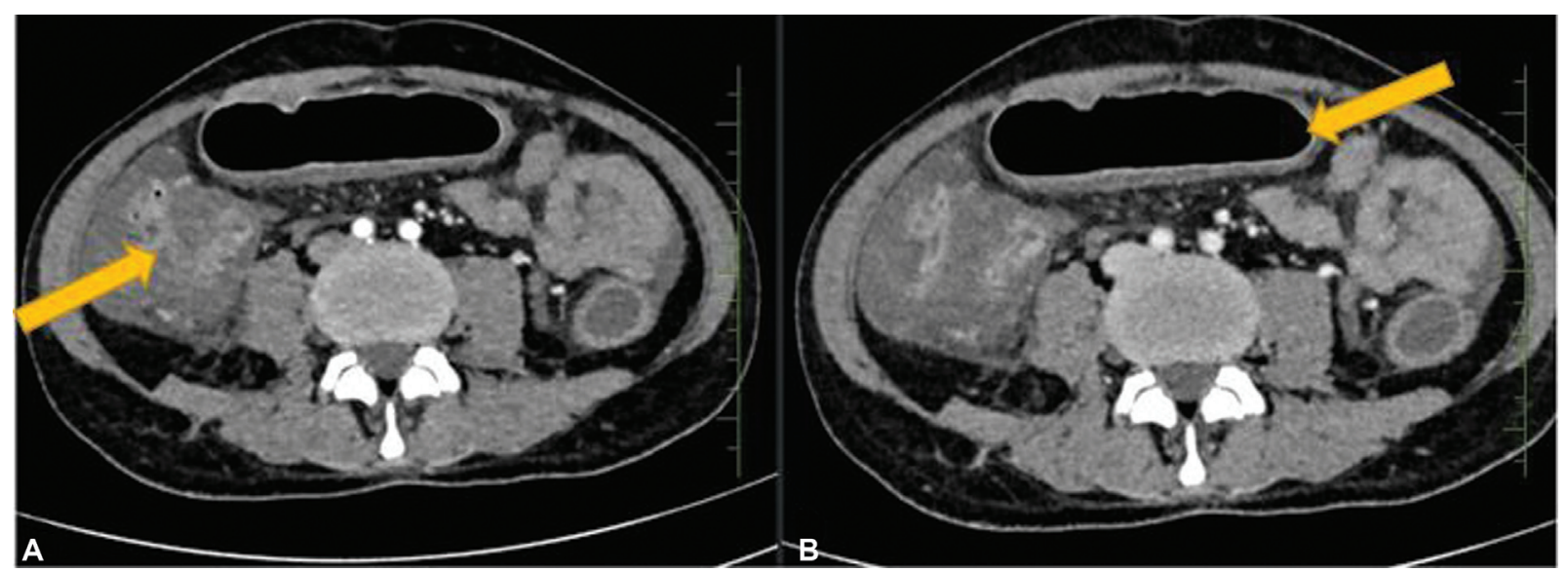

Fig. 3 (A and B) Enhanced-computed tomography axial sections of case 2 in soft tissue window reveal prominent large bowel (sigmoid loop) showing non-enhancing mucosa and pericolic fluid, indicating intestinal ischemia. 


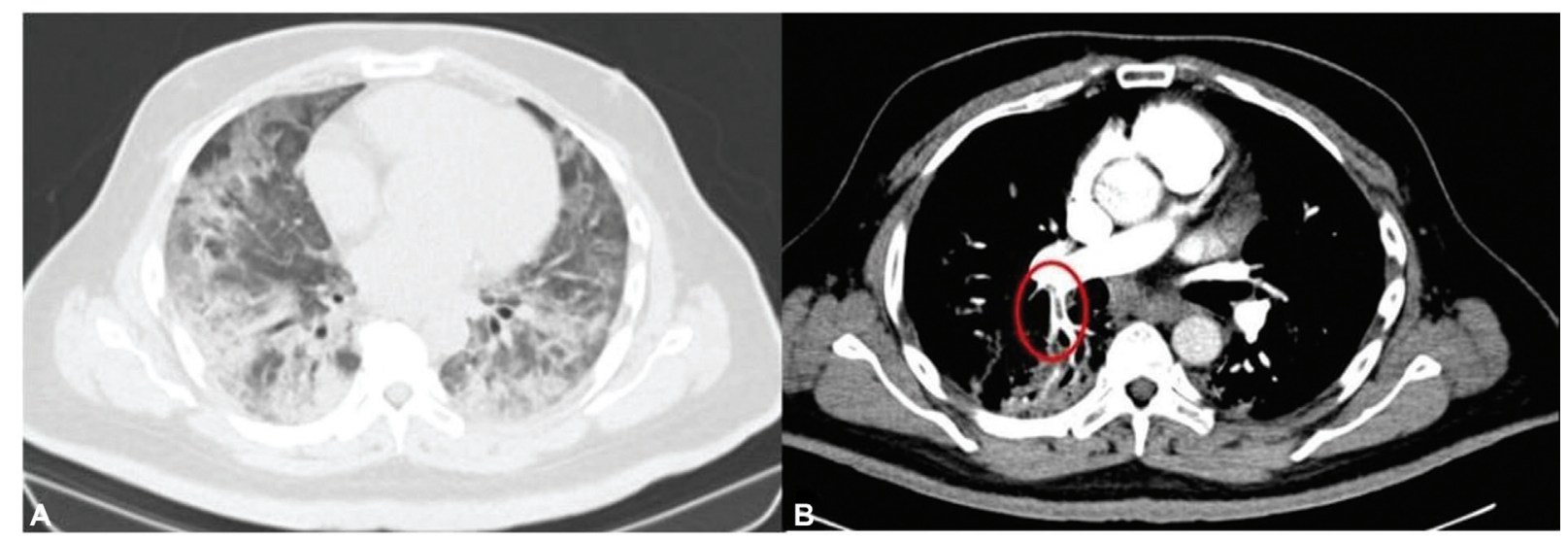

Fig. 4 Computed tomography pulmonary angiography of case 2. (A) Sub-pleural distribution of ground-glass opacities. (B) Thrombus in segmental pulmonary vessels (red circle).

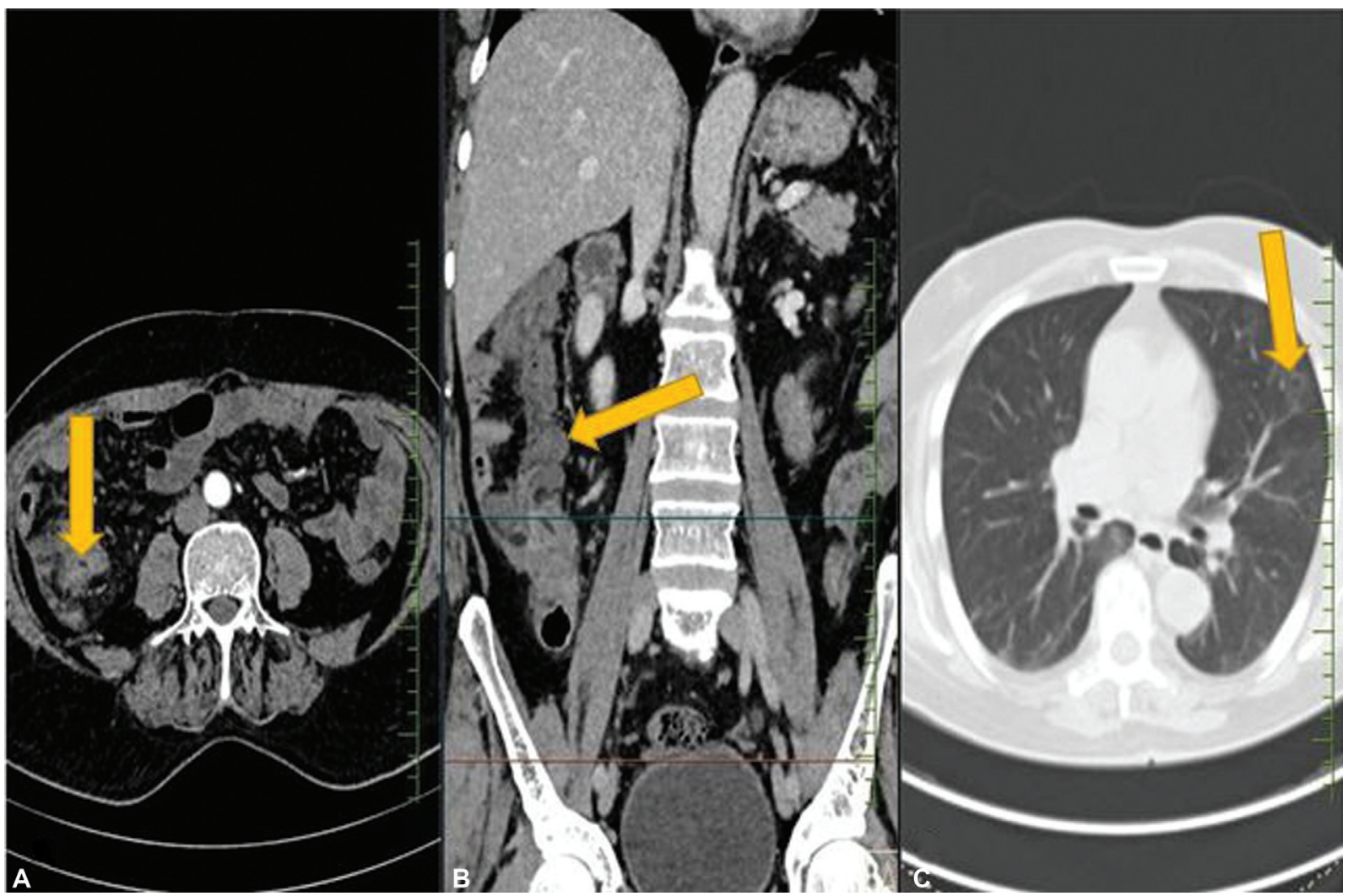

Fig. 5 (A and B) Enhanced-computed tomography axial and coronal sections of case 3 in soft tissue window reveal edematous thickening of ileocecal valve with substantial pericecal fat stranding and engorged mesentery. (C) Lower sections of lung in lung window show subtle subpleural distribution of ground-glass opacities.

\section{Discussion}

The uniqueness of the presented case series lies in the fact that all patients developed acute abdominal symptoms without any respiratory complaints. Intestinal perforation has a rare association with severe acute respiratory syndrome coronavirus 2 (SARS CoV2) infection. Case reports of perforation in SARS CoV2 patients have been reported earlier. ${ }^{5}$ The mechanism of perforation is still unknown. This virus targets the angiotensin-converting enzyme 2 (ACE-2) receptor for entry by aerosols. Along with airborne, the fecaloral transmission is under investigation. Apart from the respiratory tract, there is an abundant expression of this receptor in glandular cells of gastric, duodenal, and rectal epithelia. Not only SARS-Cov2 RNA has been found in fecal samples of patients, but also viral nucleocapsid protein has been detected in the cytoplasm of epithelia of the stomach, duodenum, and rectum. ${ }^{6}$ This affinity of the virus to the intestine's epithelial cells might play some role in the pathophysiology of perforation. Moreover, hypercoagulability is 


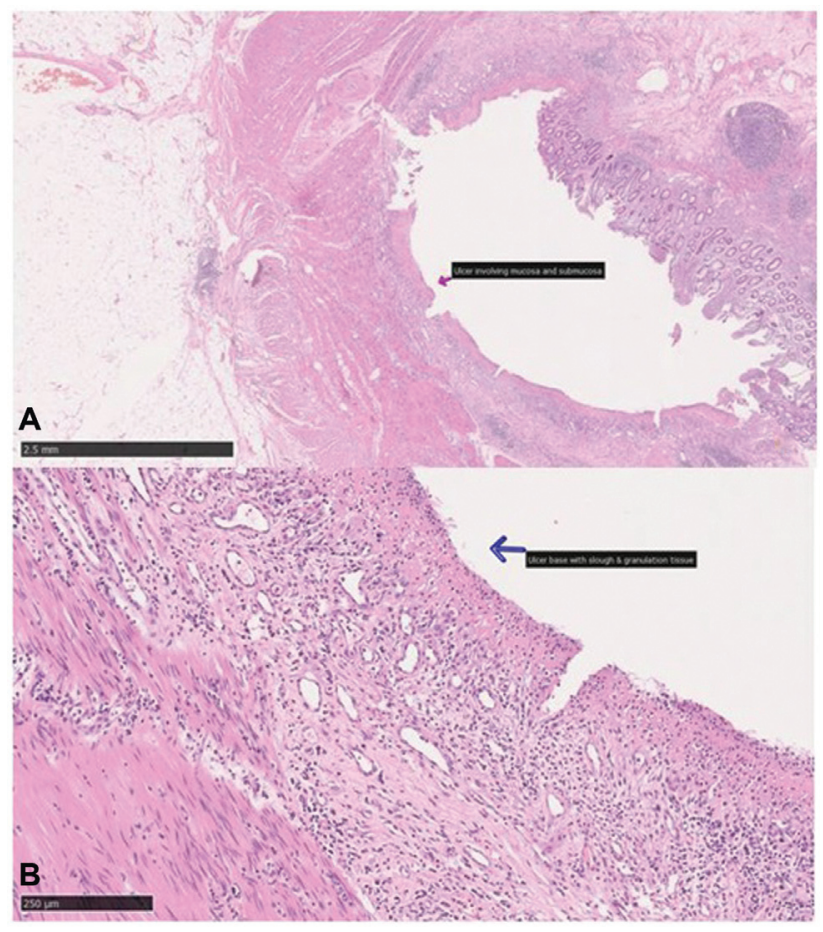

Fig. 6 (A and B) Histopathological study of case 3 done in routine hematoxylin and eosin stain and viewed under $10 \mathrm{X}$ resolution shows slough and granulation tissue on ulcer base (arrow and labeled on slide).

known to cause microcirculatory injury. ${ }^{7}$ The cytokine storm causing severe inflammatory state might play a role in mucosal injury and disseminated intravascular coagulation. Few studies have shown a similar association of bowel ischemic with colitis changes in COVID -19 infection ${ }^{8,9}$ Neurotropism of the virus leading to colonic dysmotility, distention, and bowel ischemia has also been postulated as

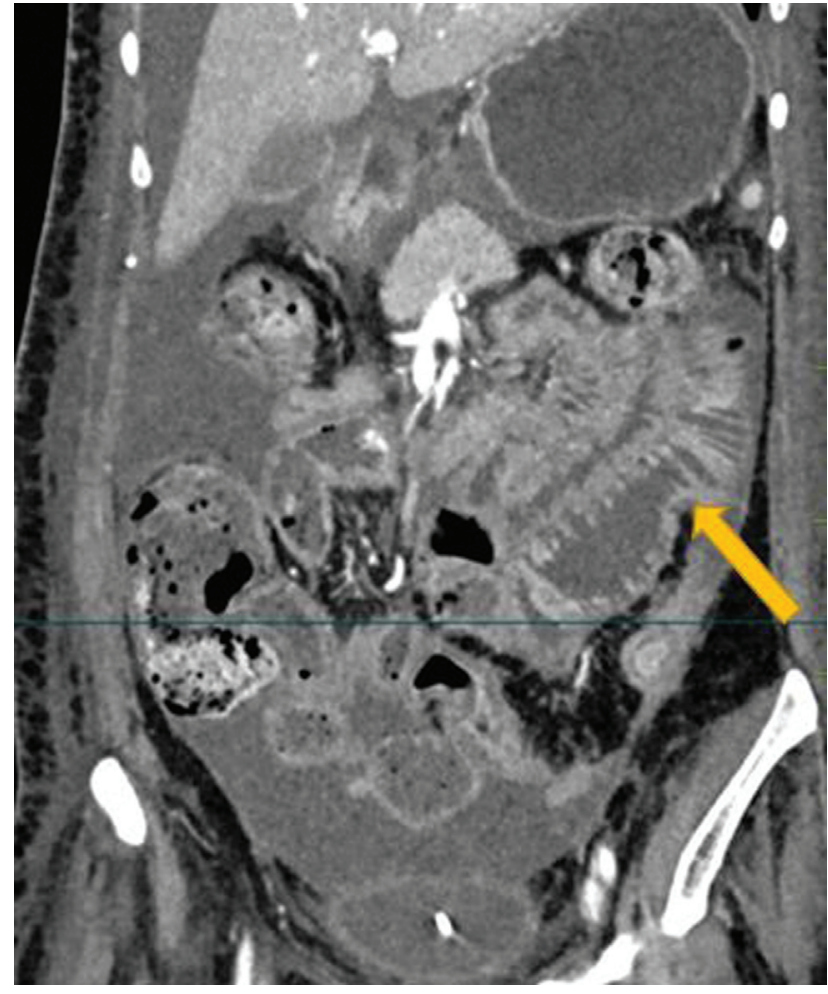

Fig. 8 Computed tomography coronal section of case 4 reveals new engorged mesentery with ascites and prominence of jejunal loops with "picket-fence" (arrow) enhancement pattern.

other possible mechanisms. ${ }^{10}$ Furthermore, increasing evidence of COVID-19 to coagulopathy ${ }^{11}$ and the state of hypercoagulability has been explained in the literature. ${ }^{12}$ The study's primary limitation was the absence of direct identification of COVID-19 RNA from GI epithelial cells; instead, only nasal swab RT-PCR verified the presence of COVID-19 infection in all cases. Even though all instances
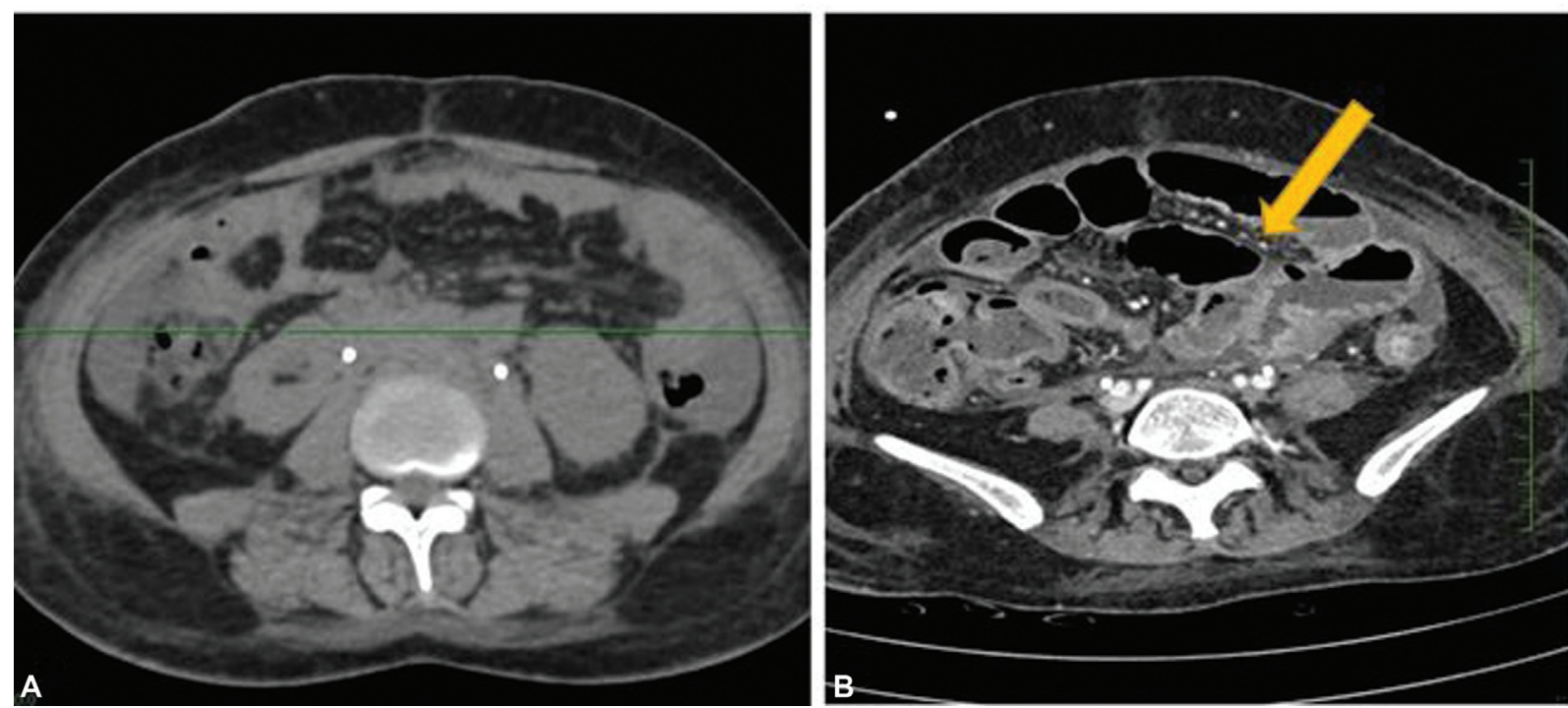

Fig. 7 (A) Computed tomography axial sections of case 4 done in July 2020 and (B) done a month later reveal new (arrow) engorged mesentery with air foci in mural walls of bowel in ileocecal junction suggestive of pneumatosis. 


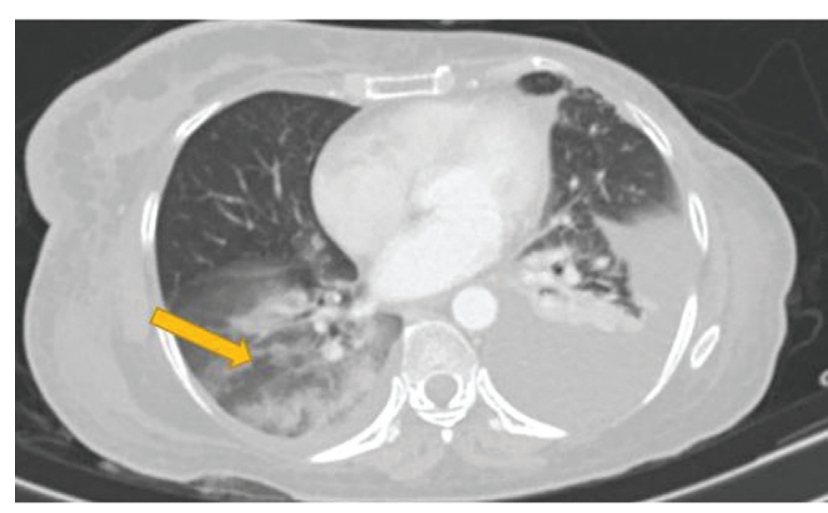

Fig. 9 Computed tomography $(\mathrm{CT})$ lower sections included in the abdominal CT of case 4 reveal pleural effusion with patchy groundglass infiltrates (arrow).

exhibited concurrent COVID infection, the precise mechanism by which the virus caused the intestinal findings recorded on CT scans in each patient could not be verified properly and directly in this investigation and had to be inferred from related and ancillary findings. However, it must be stressed that the histopathological analysis was performed in paraffin-embedded tissues since no fresh samples were obtainable. Our study highlights that perforation should be kept in the differential of SARS-Cov2 patients presenting with pain abdomen or distention. As the disease spreads, our knowledge about it grows. Having an open learning mind to the rare atypical manifestations could be beneficial in early diagnosis, and management of this underrecognized complication in this pandemic can prevent mortality as well as morbidity.

\section{What Is Already Known?}

COVID-19 patients have a higher rate of GI complications, including mesenteric ischemia. Increased expression of ACE2 receptors along the epithelial lining of the gut that acts as host-cell receptors for SARS-CoV-2 could explain the involvement of abdominal organs.

\section{What Is New in This Study?}

This case series presents unique scenarios of bowel complications suspected clinically and subsequently confirmed on imaging with COVID-19 infection. Interestingly, all patients were referred to a cancer care center where they had received previous treatment or referred for symptoms thought to be of cancerous etiology. The case series helps clinicians better understand the bowel complications in this unique subset of patients.

\section{What Are the Future Clinical and Research Implications of the Study Findings?}

As per the current procedure, the RT-PCR test for COVID-19 is performed using respiratory secretions; however, future indications may incorporate viral detection from gut wall tissue obtained via surgical specimen or tissue biopsy.

\section{Funding \\ None.}

Conflict of Interest

None declared.

\section{Acknowledgments}

The authors thank the patients and their families for their munificence in contributing to this study. They also thank all members of the Institutional Review Board committee who gave their approval for this study.

\section{References}

1 Phelan AL, Katz R, Gostin LO. The novel coronavirus originating in Wuhan, China: challenges for global health governance. JAMA 2020;323(08):709-710

2 Gao Y, Xi H, Chen L. Emergency surgery in suspected COVID-19 patients with acute abdomen: case series and perspectives. Ann Surg 2020;272(01):e38-e39

3 Rokkas T. Gastrointestinal involvement in COVID-19: a systematic review and meta-analysis. Ann Gastroenterol 2020;33(04):355-365

4 Effenberger M, Grabherr F, Mayr L, et al. Faecal calprotectin indicates intestinal inflammation in COVID-19. Gut 2020;69 (08):1543-1544

5 De Nardi P, Parolini DC, Ripa M, Racca S, Rosati R. Bowel perforation in a Covid-19 patient: case report. Int J Colorectal Dis 2020;35 (09):1797-1800

6 Xiong XL, Wong KK, Chi SQ et al. Comparative study of the clinical characteristics and epidemiological trend of 244 COVID-19 infected children with or without GI symptoms. Gut 2021;70(02):436-438

7 Zhang Y, Xiao M, Zhang S, et al. Coagulopathy and antiphospholipid antibodies in patients with Covid-19. N Engl J Med 2020;382 (17):e38

8 Bianco F, Ranieri AJ, Paterniti G, Pata F, Gallo G. Acute intestinal ischemia in a patient with COVID-19. Tech Coloproctol 2020;24 (11):1217-1218

9 Gartland RM, Velmahos GC. Bowel necrosis in the setting of COVID-19. J Gastrointest Surg 2020;24(12):2888-2889

10 Conde Cardona G, Quintana Pájaro LD, Quintero Marzola ID, Ramos Villegas Y, Moscote Salazar LR. Neurotropism of SARS-CoV 2: mechanisms and manifestations. J Neurol Sci 2020;412:116824

11 Guan WJ, Ni ZY, Hu Y, et al; China Medical Treatment Expert Group for Covid-19. Clinical characteristics of coronavirus disease 2019 in China. N Engl J Med 2020;382(18):1708-1720

12 Panigada M, Bottino N, Tagliabue P, et al. Hypercoagulability of COVID-19 patients in intensive care unit: a report of thromboelastography findings and other parameters of hemostasis. J Thromb Haemost 2020;18(07):1738-1742 